\title{
Consulta de pré-natal associada aos melhores desfechos neonatais em um estado no nordeste do Brasil: uma série histórica
}

Prenatal consultation associated with the best neonatal outcomes in a state in northeastern Brazill: a historical series

Consulta prenatal asociada a los mejores resultados neonatales en un estado del nordeste de Brasil: una serie histórica

\section{Resumo}

O objetivo deste estudo foi analisar o perfil dos nascidos vivos no Estado de Alagoas, pertencente ao nordeste do Brasil, correlacionando com o número de consultas de pré-natal no recorte temporal de dez anos, entre os anos de 2009 a 2018. Trata-se de um estudo descritivo, retrospectivo, com abordagem quantitativa, realizado através de dados do Sistema de Informação sobre Nascidos Vivos (SINASC). Os resultados demonstraram que houve uma relação positiva entre o aumento do número de consultas ao longo dos anos, com predomínio de uma maior faixa etária e maior escolaridade entre as mulheres, demonstrando que este grupo possui mais chances de acesso aos serviços de saúde. Além disso, quando comparados os melhores desfechos neonatais, demonstrou-se que mulheres com uma alta cobertura de pré-natal, apresentaram melhores resultados quanto ao índice de Apgar no $1^{\circ}$ e $5^{\circ}$ minuto e melhor peso ao nascimento. Com base nisso, embora tenha sido evidenciado um aumento no número de consultas ao longo dos anos, com melhores desfechos neonatais, esse fator não garante eficácia e qualidade na assistência quando analisado isoladamente. É necessário que gestores e profissionais da saúde assumam a responsabilidade ao cuidar de mulheres no ciclo gravídico, para uma implementação efetiva da política pública em saúde da mulher.

Palavras-chave: Cuidado pré-natal; Nascimento vivo; Perfil de saúde; Saúde pública. 


\begin{abstract}
The aim of this study was to analyze the profile of live births in the State of Alagoas, belonging to northeastern Brazil, correlating with the number of prenatal consultations in the ten-year time frame, between 2009 and 2018. This is a descriptive, retrospective study with a quantitative approach, carried out through data from the Information System on Live Births (SINASC). The results showed that there was a positive relationship between the increase in the number of consultations over the years, with a predominance of a higher age group and higher education among women, demonstrating that this group has more chances of access to health services. Moreover, when comparing the best neonatal outcomes, it was demonstrated that women with a high prenatal coverage presented better results regarding the Apgar index in the 1st and 5th minute and better birth weight. Based on this, although an increase in the number of consultations over the years, with better neonatal outcomes, has been evidenced, this factor does not guarantee efficacy and quality of care when analyzed alone. It is necessary that managers and health professionals take responsibility for caring for women in the pregnancy cycle, for an effective implementation of public policy on women's health.
\end{abstract}

Keywords: Prenatal care; Live birth; Health profile; Public health.

\title{
Resumen
}

El objetivo de este estudio fue analizar el perfil de nacidos vivos en el Estado de Alagoas, perteneciente al nordeste de Brasil, correlacionando con el número de consultas prenatales en el marco de diez años, entre 2009 y 2018 . Se trata de un estudio descriptivo, retrospectivo y de abordaje cuantitativo, realizado a partir de datos del Sistema de Información sobre Nacidos Vivos (SINASC). Los resultados mostraron que hubo una relación positiva entre el aumento en el número de consultas a lo largo de los años, con un predominio de un grupo de edad más alto y la educación superior entre las mujeres, lo que demuestra que este grupo tiene más posibilidades de acceso a los servicios de salud. Además, al comparar los mejores resultados neonatales, se demostró que las mujeres con una alta cobertura prenatal presentaron mejores resultados con respecto al índice de Apgar en el minuto 1 y 5 y mejor peso al nacer. En base a esto, aunque se ha evidenciado un aumento en el número de consultas a lo largo de los años, con mejores resultados neonatales, este factor no garantiza la eficacia y calidad de la atención cuando se analiza solo. Es necesario que los gestores y profesionales de la salud asuman la responsabilidad de cuidar a las mujeres en el ciclo del embarazo, para una implementación efectiva de la política pública en materia de salud de la mujer.

Palabras clave: Atención prenatal; Nacimiento vivo; Perfil de salud; Salud pública.

\section{Introdução}

Este estudo tem como objeto de pesquisa o perfil dos nascidos vivos associado ao número de consultas de Pré-natal no Estado de Alagoas.

A assistência ao pré-natal na sua amplitude visa reduzir as taxas de morbimortalidade materna e neonatal através de ações voltadas à saúde da mulher, realizando acompanhamento e monitorização para identificar situações de risco que possam aumentar essas taxas e a partir disso, elaborar estratégias que diminuam significativamente os desfechos perinatais negativos (Nascimento, et al., 2020; Lessa, et al., 2019).

No Brasil, com o intuito de fortalecer a cobertura de pré-natal, criou-se, pelo Ministério da Saúde, o Programa Nacional de Humanização no Pré-natal e Nascimento (PNHN), no ano de 2000, estabelecendo critérios para um acompanhamento de qualidade. Além disso, em 2011 o governo federal lançou a Rede Cegonha, cujo objetivo se associava ao PNHN, buscando-se também reduzir a mortalidade materna, sobretudo nas regiões Norte e Nordeste do país (Mario, et al., 2019).

A cobertura de assistência ao pré-natal vem crescendo desde a década de 90, atingindo valores superiores a 90\%. Porém, quando esse acesso é inexistente ou apresenta dificuldades de acompanhamento, associa-se ao aumento das taxas de mortalidade perinatal, fato que ocorre principalmente nos países subdesenvolvidos, os quais predominam óbitos perinatais por patologias preveníveis ou controladas através de um pré-natal de qualidade (Rocha, Antoneli, Leite, Ribeiro \& Terra, 2021).

Somando-se a isso, as desigualdades regionais de acesso à assistência ainda persistem, principalmente na região Nordeste, na qual se observa maior incidência de fatores relacionados a um pré-natal de baixa qualidade, como menores percentuais de cobertura, menor adesão e realização de exames e maiores taxas de mortalidade materna e neonatal (Silva, Lima \& Osório, 2019). 
Nesse contexto, o estudo se justifica pela importância da realização de pesquisas com essa temática e que possam proporcionar estratégias de enfrentamento ao pré-natal realizado inadequadamente e o aumento das chances de desfechos negativos, como por exemplo, a prematuridade, baixo peso ao nascer, mortalidades materna, fetal e neonatal, quadro de depressão puerperal e até mesmo gestações sucessivas em pouco tempo (Cunha, Lacerda, Alcauza \& Natal, 2019).

Com base nisso, o presente estudo possui como objetivo analisar o perfil dos nascidos vivos no Estado de Alagoas, pertencente ao nordeste do Brasil, correlacionando com o número de consultas de pré-natal entre os anos de 2009 a 2018.

\section{Metodologia}

Trata-se de um estudo descritivo, retrospectivo, com abordagem quantitativa, em uma epidemiologia descritiva, que segundo Lima-Costa \& Barreto (2003), são utilizados para determinar como a incidência ou prevalência de uma doença ou condição de saúde poder variar de acordo com determinadas características. Para isso, este estudo foi realizado através de dados do Sistema de Informação sobre Nascidos Vivos (SINASC), sobre o perfil dos nascidos vivos no Estado de Alagoas, Brasil, de acordo com o número de consultas de pré-natal realizadas pela genitora, na série histórica de dez anos (2009 a 2018).

Os dados do SINASC foram coletados no mês de novembro de 2020 na base de Informações de Saúde (Tabnet) do Departamento de Informática do Sistema Único de Saúde (Datasus), disponibilizada pelo Ministério da Saúde. Vale destacar que as informações contidas nesse sistema de informação são provenientes das Declarações de Nascidos Vivos do respectivo período.

Em relação ao número de consultas de pré-natal realizadas, as mesmas foram agrupadas da seguinte forma: de nenhuma a 3 consultas, de 4 a 6 consultas e de 7 ou mais consultas. Devido ao número significativo de registros sem essa informação, também foi considerado o grupo de ignorados. A partir dessa definição, as variáveis escolhidas para o estudo foram subdivididas em: sociodemográficas maternas (idade, escolaridade e estado civil), maternas relacionadas à gestação (duração da gestação, tipo de gravidez e tipo de parto) e relacionadas aos recém-nascidos (sexo, raça/cor, idade gestacional, Apgar no $1^{\circ}$ e $5^{\circ}$ minuto de vida e peso ao nascer).

Após a coleta de dados, os mesmos foram inseridos em planilha utilizando o Microsoft Excel 2016 por Windows®, analisados utilizando a estatística descritiva por frequência absoluta e relativa, sendo estes dados apresentados através de gráficos e tabelas.

Quanto aos aspectos éticos, por se tratar de um estudo utilizando como fonte os dados secundários coletados numa plataforma de domínio público, essa pesquisa não necessitou ser submetida ao Comitê de Ética em Pesquisa com seres humanos (CEP) conforme a Resolução nº 510, de 7 de abril de 2016.

\section{Resultados}

No período de 2009 a 2018, foram notificados no estado de Alagoas 524.058 nascimentos de crianças vivas, deste total, $63.748(12,2 \%)$ das gestantes realizaram de nenhuma até 3 (três) consultas de pré-natal, enquanto 193.289 (36,9\%) das genitoras realizaram 4 (quatro) a 6 (seis) consultas e $262.586(50,1 \%)$ realizaram 7 (sete) ou mais consultas.

$\mathrm{Na}$ figura 1, contendo gráfico, observa-se que ao longo do período em estudo o percentual de gestantes que realizaram 7 ou mais consultas apresentou um aumento significativo, com incremento de 42,9\%, comparando o ano inicial (2009) e final (2018), já o número de gestantes que realizaram de 0 a 3 apresentou uma variabilidade ao longo dos anos, tendendo a queda a partir de 2011, enquanto que a proporção das que realizaram de 4 a 6 consultas manteve-se em declínio. 
Figura 1: Gráfico de Evolução do percentual do número de consultas de pré-natal realizadas, segundo ano de nascimento. Alagoas, 2009-2018.

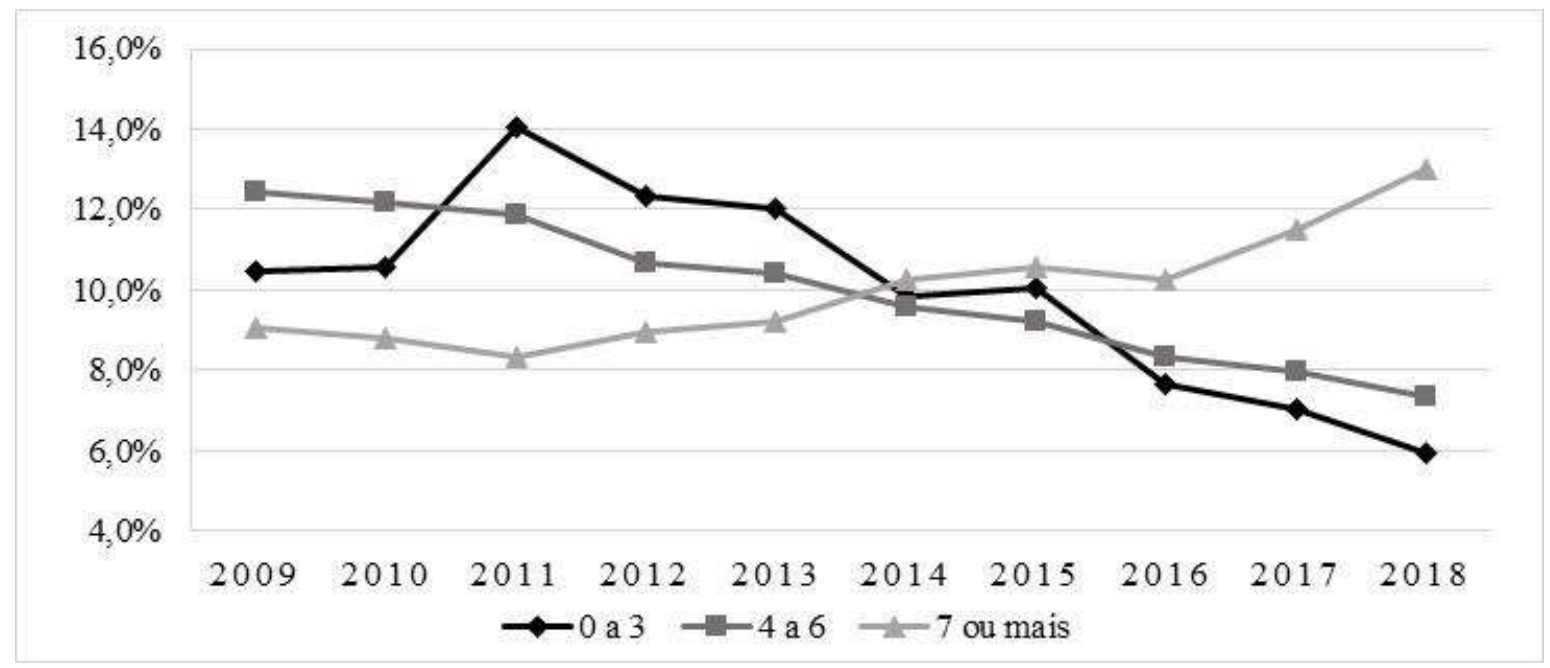

Fonte: Sistema de Informação sobre Nascidos Vivos-SINASC. Tabnet/DataSUS.

De acordo com as características sociodemográficas maternas (Tabela 1), observa-se que a faixa etária de 20 a 29 anos corresponde a maior proporção $(51,1 \%)$, seguida pela faixa etária de 10 a 19 anos $(25,3 \%)$, contudo houve pequenas diferenças estatísticas entre a idade materna e o número de consultas de pré-natal, observando-se que quanto maior a idade, maiores as chances de realizar mais consultas.

Tabela 1: Características sociodemográficas maternas segundo grupo de número de consultas de pré-natal. Alagoas, 20092018.

\begin{tabular}{|c|c|c|c|c|c|c|c|c|c|c|}
\hline \multirow[t]{2}{*}{ Variáveis } & \multicolumn{2}{|c|}{$\begin{array}{c}\text { Nenhuma a } 3 \\
\text { consultas } \\
(n=63.748)\end{array}$} & \multicolumn{2}{|c|}{$\begin{array}{c}4 \text { a } 6 \text { consultas } \\
(n=193.289)\end{array}$} & \multicolumn{2}{|c|}{$\begin{array}{c}7 \text { ou mais consultas } \\
(n=262.586)\end{array}$} & \multicolumn{2}{|c|}{$\begin{array}{l}\text { Ignorado } \\
(n=4.435)\end{array}$} & \multicolumn{2}{|c|}{$\begin{array}{c}\text { Total } \\
(\mathrm{N}=\mathbf{5 2 4 . 0 5 8})\end{array}$} \\
\hline & $\begin{array}{c}\text { número } \\
\text { absoluto }\end{array}$ & $\%$ & $\begin{array}{c}\text { número } \\
\text { absoluto }\end{array}$ & $\%$ & $\begin{array}{c}\text { número } \\
\text { absoluto }\end{array}$ & & $\begin{array}{c}\text { número } \\
\text { absoluto }\end{array}$ & $\%$ & $\begin{array}{c}\text { número } \\
\text { absoluto }\end{array}$ & $\%$ \\
\hline \multicolumn{11}{|l|}{ Idade } \\
\hline 10 a 19 anos & 19.661 & 30,8 & 55.268 & 28,6 & 56.486 & 21,5 & 1.028 & 23,2 & 132.443 & 25,3 \\
\hline 20 a 29 anos & 30.978 & 48,6 & 97.855 & 50,6 & 136.507 & 52,0 & 2.210 & 49,8 & 267.550 & 51,1 \\
\hline 30 a 39 anos & 11.648 & 18,3 & 36.678 & 19,0 & 64.808 & 24,7 & 1.054 & 23,8 & 114.188 & 21,8 \\
\hline 40 a 49 anos & 1.447 & 2,3 & 3.471 & 1,8 & 4.771 & 1,8 & 143 & 3,2 & 9.832 & 1,9 \\
\hline 50 anos ou mais & 14 & 0,02 & 16 & 0,01 & 14 & 0,01 & 0 & 0 & 44 & 0,01 \\
\hline \multicolumn{11}{|l|}{ Escolaridade } \\
\hline 0 a 3 anos & 12.616 & 19,8 & 25.947 & 13,4 & 23.015 & 8,8 & 759 & 17,1 & 62.337 & 11,9 \\
\hline 4 a 7 anos & 24.967 & 39,2 & 68.963 & 35,7 & 68.279 & 26,0 & 1.344 & 30,3 & 163.553 & 31,2 \\
\hline 8 a 11 anos & 22.074 & 34,6 & 82.938 & 42,9 & 126.523 & 48,2 & 1.478 & 33,3 & 233.013 & 44,5 \\
\hline 12 ou mais & 2.601 & 4,1 & 12.328 & 6,4 & 40.130 & 15,3 & 635 & 14,3 & 55.694 & 10,6 \\
\hline \multicolumn{11}{|l|}{ Estado Civil } \\
\hline Solteira & 31.772 & 49,8 & 84.885 & 43,9 & 87.026 & 33,1 & 2.058 & 46,4 & 205.741 & 39,3 \\
\hline Casada & 11.900 & 18,7 & 48.891 & 25,3 & 96.757 & 36,8 & 1.249 & 28,2 & 158.797 & 30,3 \\
\hline Viúva & 149 & 0,2 & 385 & 0,2 & 476 & 0,2 & 11 & 0,2 & 1.021 & 0,2 \\
\hline Separada & 216 & 0,3 & 681 & 0,4 & 1.343 & 0,5 & 25 & 0,6 & 2.265 & 0,4 \\
\hline União consensual & 18.332 & 28,8 & 55.515 & 28,7 & 73.831 & 28,1 & 924 & 20,8 & 148.602 & 28,4 \\
\hline
\end{tabular}


Em relação à escolaridade, percebe-se que quanto maior o tempo de estudos, maior foi o número de consultas realizadas. Ao analisar o estado civil, as mulheres solteiras (39,3\%) realizaram menos consultas de pré-natal comparado as mulheres casadas $(30,3 \%)$.

Ao analisar as características gestacionais segundo grupo de número de consultas de pré-natal (Tabela 2), observou-se que o número de consultas de pré-natal é maior à medida que a duração da gestação também é maior. Em relação ao tipo de gestação, observou-se que não há diferença percentual significativa entre os grupos, mas em números absolutos percebe-se maior quantidade de consultas de pré-natal em gestações gemelares. Nessa tabela, cabe atentar-se que quanto maior o número de consultas de pré-natal, maior o percentual de partos cesáreos - 60,8\% das mulheres que fizeram cesarianas possuíam 7 ou mais consultas de pré-natal, enquanto que $61,1 \%$ das mulheres que tiveram parto vaginal possuíam 0 a 3 consultas.

Tabela 2: Características maternas relacionadas à gestação, segundo grupo de número de consultas de pré-natal. Alagoas, 2009-2018.

\begin{tabular}{|c|c|c|c|c|c|c|c|c|c|c|}
\hline \multirow[t]{2}{*}{ Variáveis } & \multicolumn{2}{|c|}{$\begin{array}{c}\text { Nenhuma a } 3 \\
\text { consultas } \\
(n=63.748)\end{array}$} & \multicolumn{2}{|c|}{$\begin{array}{c}4 \text { a } 6 \text { consultas } \\
(n=193.289)\end{array}$} & \multicolumn{2}{|c|}{$\begin{array}{l}7 \text { ou mais consultas } \\
\quad(n=262.586)\end{array}$} & \multicolumn{2}{|c|}{$\begin{array}{l}\text { Ignorado } \\
(n=4.435)\end{array}$} & \multicolumn{2}{|c|}{$\begin{array}{c}\text { Total } \\
(\mathrm{N}=\mathbf{5 2 4 . 0 5 8})\end{array}$} \\
\hline & $\begin{array}{l}\text { número } \\
\text { absoluto }\end{array}$ & $\%$ & $\begin{array}{l}\text { número } \\
\text { absoluto }\end{array}$ & $\%$ & $\begin{array}{l}\text { número } \\
\text { absoluto }\end{array}$ & $\%$ & $\begin{array}{l}\text { número } \\
\text { absoluto }\end{array}$ & $\%$ & $\begin{array}{l}\text { número } \\
\text { absoluto }\end{array}$ & $\%$ \\
\hline \multicolumn{11}{|l|}{$\begin{array}{l}\text { Duração da } \\
\text { gestação }\end{array}$} \\
\hline$\leq 36$ semanas & 10.085 & 15,8 & 22.500 & 11,6 & 17.018 & 6,5 & 626 & 14,1 & 50.229 & 9,6 \\
\hline $\begin{array}{l}37 \text { a } 41 \\
\text { semanas }\end{array}$ & 39.707 & 62,3 & 153.345 & 79,3 & 221.592 & 84,4 & 3.263 & 73,6 & 417.907 & 79,7 \\
\hline$\geq 42$ semanas & 1.725 & 2,7 & 6.579 & 3,4 & 11.174 & 4,3 & 60 & 1,4 & 19.538 & 3,7 \\
\hline Ignorado & 12.231 & 19,2 & 10.865 & 5,6 & 12.802 & 4,9 & 486 & 11,0 & 36.384 & 6,9 \\
\hline \multicolumn{11}{|l|}{ Tipo de } \\
\hline Única & 61.966 & 97,2 & 189.784 & 98,2 & 258.335 & 98,4 & 4.250 & 95,8 & 514.335 & 98,1 \\
\hline Dupla & 1.284 & 2,0 & 3.193 & 1,7 & 3.859 & 1,5 & 148 & 3,3 & 8.484 & 1,6 \\
\hline $\begin{array}{l}\text { Tripla ou } \\
\text { mais }\end{array}$ & 47 & 0,1 & 62 & 0,03 & 70 & 0,03 & 4 & 0,1 & 183 & 0,03 \\
\hline Ignorado & 451 & 0,7 & 250 & 0,1 & 322 & 0,1 & 33 & 0,7 & 1.056 & 0,2 \\
\hline \multicolumn{11}{|l|}{$\begin{array}{l}\text { Tipo de } \\
\text { parto }\end{array}$} \\
\hline Vaginal & 38.947 & 61,1 & 97.660 & 50,5 & 102.653 & 39,1 & 1.970 & 44,4 & 241.230 & 46,0 \\
\hline Cesário & 24.421 & 38,3 & 95.319 & 49,3 & 159.598 & 60,8 & 2.437 & 54,9 & 281.775 & 53,8 \\
\hline Ignorado & 380 & 0,6 & 310 & 0,2 & 335 & 0,1 & 28 & 0,6 & 1.053 & 0,2 \\
\hline
\end{tabular}

Fonte: Sistema de Informação sobre Nascidos Vivos-SINASC. Tabnet/DataSUS.

Em relação às características dos recém-nascidos, observa-se que quanto maior o número de consultas de pré-natal, maior o score do índice de Apgar, tanto no $1^{\circ}$ quanto no $5^{\circ}$ minuto de vida, além disso, o peso ao nascer também é maior de acordo com o número de consultas, conforme descrito na Tabela 3. 
Tabela 3: Características dos recém-nascidos, segundo grupo de número de consultas de pré-natal. Alagoas, 2009-2018.

\begin{tabular}{|c|c|c|c|c|c|c|c|c|c|c|}
\hline \multirow[t]{2}{*}{ Variáveis } & \multicolumn{2}{|c|}{$\begin{array}{c}\text { Nenhuma a } 3 \\
\text { consultas } \\
(n=63.748)\end{array}$} & \multicolumn{2}{|c|}{$\begin{array}{c}4 \text { a } 6 \text { consultas } \\
(n=193.289)\end{array}$} & \multicolumn{2}{|c|}{$\begin{array}{c}7 \text { ou mais consultas } \\
\quad(n=262.586)\end{array}$} & \multicolumn{2}{|c|}{$\begin{array}{l}\text { Ignorado } \\
(n=4.435)\end{array}$} & \multicolumn{2}{|c|}{$\begin{array}{c}\text { Total } \\
(\mathrm{N}=\mathbf{5 2 4 . 0 5 8})\end{array}$} \\
\hline & $\begin{array}{l}\text { número } \\
\text { absoluto }\end{array}$ & $\%$ & $\begin{array}{l}\text { número } \\
\text { absoluto }\end{array}$ & $\%$ & $\begin{array}{l}\text { número } \\
\text { absoluto }\end{array}$ & $\%$ & $\begin{array}{l}\text { número } \\
\text { absoluto }\end{array}$ & $\%$ & $\begin{array}{l}\text { número } \\
\text { absoluto }\end{array}$ & $\%$ \\
\hline \multicolumn{11}{|l|}{ Sexo } \\
\hline Masculino & 32.775 & 51,4 & 99.042 & 51,2 & 134.169 & 51,1 & 2.243 & 50,6 & 268.209 & 51,2 \\
\hline Feminino & 30.970 & 48,6 & 94.198 & 48,7 & 128.380 & 48,9 & 2.191 & 49,4 & 255.739 & 48,8 \\
\hline Ignorado & 23 & 0,04 & 49 & 0,03 & 37 & 0,01 & 1 & 0,02 & 110 & 0,02 \\
\hline \multicolumn{11}{|l|}{ Raça/Cor } \\
\hline Branca & 3.995 & 6,3 & 13.268 & 6,9 & 27.085 & 10,3 & 581 & 13,1 & 44.929 & 8,6 \\
\hline Preta & 944 & 1,5 & 2.177 & 1,1 & 2.539 & 1,0 & 51 & 1,1 & 5.711 & 1,1 \\
\hline Parda & 55.673 & 87,3 & 171.098 & 88,5 & 223.251 & 85,0 & 3.660 & 82,5 & 453.682 & 86,6 \\
\hline Outra & 278 & 0,4 & 798 & 0,4 & 1.307 & 0,5 & 16 & 0,4 & 2.399 & 0,5 \\
\hline Ignorada & 2.858 & 4,5 & 5.948 & 3,1 & 8.404 & 3,2 & 127 & 2,9 & 17.337 & 3,3 \\
\hline \multicolumn{11}{|l|}{$\begin{array}{l}\text { Apgar no } 1^{\circ} \\
\text { minuto }\end{array}$} \\
\hline 0 a 7 & 8.533 & 13,4 & 24.769 & 12,8 & 28.907 & 11,0 & 777 & 17,5 & 62.986 & 12,0 \\
\hline 8 a 10 & 49.201 & 77,2 & 154.820 & 80,1 & 221.946 & 84,5 & 3.289 & 74,2 & 429.256 & 81,9 \\
\hline Ignorado & 6.014 & 9,4 & 13.700 & 7,1 & 11.733 & 4,5 & 369 & 8,3 & 31.816 & 6,1 \\
\hline \multicolumn{11}{|l|}{$\begin{array}{l}\text { Apgar no } 5^{\circ} \\
\text { minuto }\end{array}$} \\
\hline 0 a 7 & 1.980 & 3,1 & 3.672 & 1,9 & 3.592 & 1,4 & 194 & 4,4 & 9.438 & 1,8 \\
\hline 8 a 10 & 55.485 & 87,0 & 175.383 & 90,7 & 246.738 & 94,0 & 3.854 & 86,9 & 481.460 & 91,9 \\
\hline Ignorado & 6.283 & 9,9 & 14.234 & 7,4 & 12.256 & 4,7 & 387 & 8,7 & 33.160 & 6,3 \\
\hline \multicolumn{11}{|l|}{$\begin{array}{l}\text { Peso ao } \\
\text { nascer }\end{array}$} \\
\hline Até $2.499 \mathrm{~g}$ & 8.995 & 14,1 & 16.595 & 8,6 & 14.147 & 5,4 & 755 & 17,0 & 40.492 & 7,7 \\
\hline $\begin{array}{l}2.500 \mathrm{~g} \mathrm{a} \\
3.999 \mathrm{~g}\end{array}$ & 51.633 & 81,0 & 166.094 & 85,9 & 231.780 & 88,3 & 3.458 & 78,0 & 452.965 & 86 \\
\hline $4.000 \mathrm{~g}$ e mais & 3.017 & 4,7 & 10.519 & 5,4 & 16.632 & 6,3 & 209 & 4,7 & 30.377 & 5,8 \\
\hline Ignorado & 103 & 0,2 & 81 & 0,04 & 27 & 0,01 & 13 & 0,3 & 224 & 0,04 \\
\hline
\end{tabular}

Fonte: Sistema de Informação sobre Nascidos Vivos-SINASC. Tabnet/DataSUS.

\section{Discussão}

Os resultados do estudo demonstraram que, assim como evidenciado por Castro et.al (2020) e Camargos, Lemos, Martins e Felisbino-Mendes (2021), houve uma proporção satisfatória de gestantes que realizaram número superior ao mínimo de 6 consultas, conforme preconizado pelo Ministério Saúde (Brasil, 2013). No entanto, apesar de ser um dado importante para analisar a cobertura do serviço, o número de consultas de pré-natal isoladamente não reflete a qualidade da assistência prestada, uma vez que, no Brasil, ainda são elevadas as taxas de mortalidade materna e perinatal (Mattei \& Carreno, 2017; Sehnem, Saldanha, Airboit, Ribeiro, \& Paula, 2019). Nessa perspectiva, Sehnem et al. (2019) abordam que os profissionais devem considerar a consulta de pré-natal como uma estratégia de educação em saúde, de modo a torná-la uma oportunidade de estreitar vínculos e conhecer as necessidades de cada família.

Quanto às características sociodemográficas maternas, observou-se que houve aumento do número de consultas, sobretudo entre as mulheres com faixa etária de 20 a 29 anos, havendo também relação diretamente proporcional entre escolaridade e número de consultas, cuja maioria era solteira. Neste ponto, Teixeira, Carvalho, Rocha, Pereira e Enders (2018) apontam que há uma alta prevalência de nascimentos prematuros quando associados à gravidez na faixa etária da adolescência, havendo também um baixo nível de escolaridade de mães de bebês prematuros e de termo, independente da faixa etária, tornando-se, um fator relevante para agravantes no desenvolvimento fetal quando considerados os extremos de idades (Costa, et al., 2018). 
Segundo Carvalho e Oliveira, (2020), a gravidez na adolescência determina um maior risco para o binômio mãe-filho, sendo necessário priorizar as ações de saúde para esse grupo em virtude do baixo acesso aos serviços e escassez de políticas públicas.

Sobre as características maternas relacionadas à gestação, evidenciou-se que quanto maior a idade gestacional, maior o número de consultas, com aumento do mesmo, sobretudo em gestações gemelares, corroborando com os resultados demonstrados por Souza, Tanoshi e Pelloso (2019), que acrescentam que o número de consultas pode proporcionar uma redução da predisposição a anomalias fetais, quando associados à qualidade no atendimento.

Quanto ao tipo de parto, estudo realizado pelos autores supracitados, também verificou que quanto maior o número de consultas, maior o desfecho de cesarianas. Em contrapartida, Ferrari, Carvalhaes e Parada (2016) demonstraram associação entre cesárea eletiva e fatores sociodemográficos, como idade superior a 18 anos, com escolaridade de ensino médio e que exerciam trabalho remunerado; além de associação com características como: gravidez planejada, mais de sete consultas de pré-natal e parto realizado pelo Sistema Único de Saúde (SUS).

Quando se analisou essa série histórica de dez anos, observa-se uma proporção de 53,8\% de cesarianas, valor muito acima do ideal, segundo a OMS, que preconiza entre 10 a 15\% para o total da população. Situação preocupante, tendo em vista o aumento dos riscos para saúde materna (como hemorragias, embolismo pulmonar, infecções puerperais e ainda mortalidade dez vezes maior que em parto vaginal) e neonatal (como prematuridade iatrogênica, problemas respiratórios devido à imaturidade pulmonar, icterícia, aumento da mortalidade e do risco para complicações que necessitem de internação em Unidade de Terapia Intensiva Neonatal) (Silva, Costa, Almeida, Araújo, \& Amâncio, 2020; Barros, et al., 2019).

Estudo realizado por Gonçalves et al. (2016), evidenciou que apesar da produtividade, ou seja, mulheres com alta cobertura de pré-natal não receberam orientações para o parto, considerado um componente importante na eficácia e qualidade da assistência. É preciso que os profissionais exerçam o papel de educadores e orientem quanto ao trabalho de parto e benefícios do parto normal na assistência ao pré-natal.

O parto vaginal possui inúmeros benefícios tanto para mãe quanto para o bebê, como melhor recuperação materna e permite compressão pulmonar no recém-nascido, facilitando a respiração extrauterina; enquanto que o parto cesáreo está associado à maior morbimortalidade materna e maior risco perinatal (Ferrari, Magalhaes \& Parada, 2016).

Em relação às características neonatais, observou-se que quanto maior o número de consultas, maiores os índices de Apgar no $1^{\circ}$ e $5^{\circ}$ minutos e maior o peso ao nascimento. Nascimento et. al (2017), evidenciaram diferença estatística significativa, quando comparou-se o número de consultas aos desfechos neonatais negativos, no qual a razão de chances indicou maior chance de prematuridade, baixo peso e óbito neonatal em gestantes com $\leq 6$ consultas. Esses desfechos configuram-se determinantes da morbimortalidade neonatal principalmente nos países em desenvolvimento (COSTA, et al., 2018).

Ainda segundo Costa et. al (2018), os valores do Apgar no $1^{\circ}$ e $5^{\circ}$ minutos estão associados à vitalidade neonatal, refletindo também a eficácia dos serviços de assistência à gestação e ao pós-parto, e, ressalta que valores de Apgar $>7$ no $5^{\circ}$ minuto foi essencial para evolução da alta do recém-nascido.

O peso ao nascimento é influenciado por fatores como nível socioeconômico, nutrição, tabagismo e morbidade durante a gestação, sendo determinante para o estado de saúde do recém-nascido, ou seja, mulheres com nutrição inadequada e de baixo nível socioeconômico pode comprometer o crescimento e desenvolvimento fetal (Teixeira, Carvalho, Rocha, Pereira $\&$ Enders, 2018).

O número de consultas de pré-natal pode estar associado a uma série de fatores e desencadear desfechos na saúde materna, fetal e neonatal. Iniciar o pré-natal tardiamente interfere no seguimento das recomendações nacionais, 
impossibilitando a identificação precoce de alterações fetais e adoção de medidas em tempo oportuno para que a assistência materno-fetal possa ser garantida na sua qualidade e efetividade para o bem-estar da família que planeja ou não sua gestação.

\section{Conclusão}

Evidenciou-se que mulheres de maior faixa etária e com maior escolaridade têm mais chances de acesso aos serviços de saúde para consultas de pré-natal e, embora haja um aumento significativo nesse número de consultas ao longo dos anos, este fator quando analisado isoladamente, não garante eficácia e qualidade de assistência no contexto dos desfechos maternoneonatal. Apesar disso, as mulheres com uma melhor cobertura apresentaram melhores desfechos neonatais quando observados os índices de Apgar no $1^{\circ}$ e 5 minutos de vida, e melhor peso ao nascimento.

No entanto, por se tratar de uma pesquisa com dados secundários, utilizando-se os dados do SINASC, esta pesquisa possui como limitações o fato de não conhecer a realidade obstétrica materna, como história clínica e hábitos alimentares e de vida. Nesse sentido, sugere-se que novas pesquisas possam ser realizadas para confrontar as informações registradas nos sistemas de saúde, e assim, conhecer a realidade dos grupos, visando à adequação do pré-natal em sua amplitude, não somente em termos de cobertura, a fim de agregar conhecimento tendo em vista políticas que sejam capazes de modificar a realidade existente.

A implementação das políticas públicas somente se efetiva quando a gestão e profissionais assumem a responsabilidade de cuidar das mulheres grávidas, orientando-as assertivamente e realizando busca ativa para captação precoce sempre que necessário.

\section{Referências}

Barros, A. J. D. Victora, C. G. Horta, B. L. Wehrmeister, F. C. Bassani, D. Silveira, M. F. Santos, L. P. Blumenberg, C. Barros, F. C. \& Pelotas Cohorts Study Group. (2019). Antenatal care and caesarean sections: trends and inequalities in four population-based birth cohorts in Pelotas, Brazil, 1982-2015. Int $J$ Epidemiol. [internet] 48 Suppl 1: i37-i45. https://academic.oup.com/ije/article/48/Supplement_1/i37/5382483 doi:10.1093/ije/dyy211.

Brasil. Ministério da Saúde. (2013). Secretaria de Atenção à Saúde. Departamento de Atenção Básica. Atenção ao pré-natal de baixo risco [recurso eletrônico] / Ministério da Saúde. Secretaria de Atenção à Saúde. Departamento de Atenção Básica. Editora do Ministério da Saúde.

Camargos, L. F. Lemos, P. L. Martins, E. F. \& Felisbino-Mendes, M. S. (2021). Avaliação da qualidade dos registros de cartões de pré-natal de mulheres urbanas. Escola Anna Nery. Vol. 25, n. 1. https://doi.org/10.1590/2177-9465-EAN-2020-0166.

Carvalho, S. S. \& Oliveira, L. F. (2020). Percepção de adolescentes gestantes sobre a assistência de enfermagem ao pré-natal. Enferm. Foco. 11 (3): $195-201$.

Castro, L. L. S. Oliveira, I. G. Bezerra, R. A. Souza, L. B. Anjos, S. J. S. B. \& Santos, L. V. F. (2020). Assistência pré-natal segundo registros profissionais presentes na caderneta da gestante. Revista de enfermagem da UFSM. 10, 1-18. 10.5902/2179769231236.

Correio, R. A. S. Correio, L. F. \& Correio, M. A. B. (2016). Perfil epidemiológico dos nascidos vivos no município de Chapecó-SC. RECIIS - Rev Eletron Comun Inf Inov Saúde. 10(2).

Costa, L. D. Freitas, P. C. Teixeira, G. T. Costa, G. Viana, V. \& Schiavoni, D. (2018). Impacto das características maternas e perinatais na evolução do recémnascido. Rev Enferm UFSM. 8(2): 334-349. 10.5902/2179769230243.

Cunha, A. C. Lacerda, J. T. Alcauza, M. T. R. \& Natal, S. (2019). Avaliação da atenção ao pré-natal na Atenção Básica no Brasil. Rev. Bras. Saúde Mater. Infant. 19(2):459-470. https://www.scielo.br/scielo.php?script=sci_arttext\&amp;pid=S1519-38292019000200447\&amp;tlng=en 10.1590/180693042019000200011 .

Ferrari, A. P. Carvalhaes, M. A. B. L. \& Parada, C. M. G. L. (2016, Jan-Mar). Associação entre pré-natal e parto na rede de saúde suplementar e cesárea eletiva. Rev Bras Epidemiol.; 19(1): 75-88. 10.1590/1980-5497201600010007.

Gonçalves, M. F. Teixeira, E. M. B. Silva, M. A. S. Corsi, N. M. Ferrari, R. A. P. Pelloso, S. M. \& Cardelli, A. A. M. (2017). Pré-natal: preparo para o parto na atenção primária a saúde no sul do Brasil. Rev Gaucha Enferm. 38(3):e2016-0063. http://dx.doi.org/10.1590/1983-1447.2017.03.2016-0063.

Lessa, M. S. A. Nascimento, E. R. Coelho, E. A. C. Nunes, I. M. Soares, I. J. \& Rodrigues, Q. P. (2019). Adequação do pré-natal no Brasil e associação com raça / cor: estudo transversal. Online braz. j. nurs. 18(1). https://doi.org/10.17665/1676-4285.20196192.

Lima-Costa, M. F. \& Barreto, S. M. (2003). Tipos de estudos epidemiológicos: conceitos básicos e aplicações na área do envelhecimento. Epidemiologia e Serviços de Saúde. 12(4), 189 - 201. < http://scielo.iec.gov.br/pdf/ess/v12n4/v12n4a03.pdf>. 
Research, Society and Development, v. 10, n. 15, e298101522601, 2021

(CC BY 4.0) | ISSN 2525-3409 | DOI: http://dx.doi.org/10.33448/rsd-v10i15.22601

Mario, D. N. Rigo, L. Boclin, K. L. S. Malvestio, L. M. M. Anziliero, D. Horta, B. L. Wehrmeister, F. C. \& Martínez-Mesa, J. (2019). Qualidade do PréNatal no Brasil: Pesquisa Nacional de Saúde 2013. Ciênc. Saúde Colet; 24(3). 1223-1232. 10.1590/1413-81232018243.131220171.

Mattei, F. \& Carreno, I. Fatores associados à saúde materno-infantil no Rio Grande do Sul, Brasil. (2017) Rev. Bras. Saúde Matern. Infantil. Recife, 17 (3): 539-549. http://dx.doi.org/10.1590/1806-93042017000300007.

Nascimento, I. B. Pacheco, V. C. Souza, M. L. R. Pinheiro, E. B. Silva, T. R. Fleig, R. \& Silva, J. C. (2017). Assistência pré-natal e resultado perinatal. Rev Bras Promoç Saúde, Fortaleza, 30(2): 187-194. 10.5020/18061230.2017.p187.

Nascimento, L. C. S. Silva, M. R. F. Abreu, P. D. Araújo, E. C. Menezes, M. L. N. \& Oliveira, E. C. T. (2020). Perspectiva dos enfermeiros sobre a assistência pré-natal no âmbito da Estratégia Saúde da Família. Rev. Enferm. UFSM - REUFSM. 10, 1-21. 10.5902/2179769238444

Rocha, S. N. Antoneli, S. O. Leite, E. P. R. C. Ribeiro, P. M. \& Terra, F. S. (2021, jan/dez). Dificuldades enfrentadas pelos enfermeiros para a realização das consultas de pré-natal de risco habitual. R. pesq.: cuid. fundam. Online. 13:966-973. http://dx.doi.org/0.9789/2175-5361.rpcfo.v13.9738.

Sehnem, G. D. Saldanha, L. S. Airboit, J. Ribeiro, A. C. \& Paula, F. M. (2019). Consulta de pré-natal na atenção primária à saúde: fragilidades e potencialidades da intervenção de enfermeiros brasileiros. Revista de Enfermagem Referência. 5(1), e19050. 10.12707/RIV19050.

Silva, E. P. Leite, A. L. B. Lima, R. T. \& Osório, M. M. (2019). Avaliação do pré-natal na atenção primária no Nordeste do Brasil: fatores associados à sua adequação. Rev Saude Publica. 53:43. https://doi.org/10.11606/S1518-8787.2019053001024.

Silva, E. V. Costa, M. A. A. Almeida, K. C. Araújo, L. M. B. \& Amâncio, N. F. G. (2020). Relação do tipo de parto com o perfil epidemiológico da assistência pré-natal e perinatal em um município de Minas Gerais. Rev. Bras. Saúde Mater. Infant. [internet]. 20(1):249-256. https://www.scielo.br/scielo.php?script=sci_arttext\&amp;pid=S1519-38292020000100241\&amp;tlng=en 10.1590/1806-93042020000100013.

Souza, L. S. Tanoshi, C. A. \& Pelloso, S. M. (2019). Tipo de parto e sua correlação com o número de consultas de Pré-natal e idade materna nas diversas regiões do brasil. Anais $28^{\circ}$ encontro anual de iniciação científica. www.eaic.uem.br/eaic2019/anais/artigos/3842.pdf.

Teixeira, G. A. Carvalho, J. B. L. Rocha, B. G. Pereira, S. A. \& Enders, B. C. (2018). Perfil de mães e o desfecho do nascimento prematuro ou a termo. Cogitare Enferm. (23)1: e51409. http://dx.doi.org/10.5380/ce.v23i1.51409. 